\title{
Testing Program for Security using Symbolic Execution and Exception Injection
}

\author{
Faisal Anwer ${ }^{1 *}$, Mohd. Nazir ${ }^{2}$ and Khurram Mustafa ${ }^{2}$ \\ 1Department of Computer Science, Aligarh Muslim University, Aligarh, India; faisalanwer.cs@amu.ac.in \\ 2Department of Computer Science, Jamia Millia Islamia (A Central University), Jamia Nagar, New Delhi, India; \\ mnazir@jmi.ac.in, kmustafa@jmi.ac.in
}

\begin{abstract}
Objective: Symbolic execution is one of the most popular automated testing techniques for program verification and test case generation. It assures exhaustive path coverage by generating and resolving path constraints on each branch, effectively covering untested paths or gray areas that often lead to security vulnerabilities. Our main objective here is to propose a testing framework to test security vulnerabilities raised due to improper error handling such as resource leakage, program crash and program inconsistency. Methods: Authors propose a framework Symexc that tests and verifies if such vulnerabilities exist in the program using symbolic execution and exception injection. Conclusion: In this paper, symbolic execution has been adopted to traverse each and every path to execute different pre-injected exceptions. The paper demonstrates the implementation of the framework, followed by its validation on some java programs. Applications: Finding these vulnerabilities during application development will check misuse of applications and hence will add more quality to the application.
\end{abstract}

Keywords: Denial of Service, Improper Error Handling, Program Crash, Program Inconsistency, Resource Leakage, Symbolic Execution

\section{Introduction}

Currently software companies are facing serious challenges in developing and testing their products. Size of software is growing rapidly, consequently the program code is becoming increasingly complicated which is making software more complex ${ }^{1}$. Missing secure coding practices may lead to several security vulnerabilities in applications either desktop or $\mathrm{web}^{2,3}$. A number of such vulnerabilities may be caused by improper error handling that raises several security issues, which prominently includes resource leakage, program crash, and program inconsistency. Recently $\mathrm{HP}^{4}$ reported that $93.87 \%$ of 359 unique web applications were found vulnerable to information leakage and improper error handling when these were analyzed through static analysis while dynamic analysis of a sample of 2,174 unique web applications showed that $15 \%$ of those were vulnerable to improper error handling and information leakage. These vulnerabilities may be exploited by attackers easily, that may result in the severe setback to applications.

Developing secure software has always been a challenging task and yet an indispensable aspect in present scenario and future development depends a lot on how we are going to tackle it. Software security ensures that software application will not remain an easy prey for an attacker. Unfortunately Security testing is rarely being practiced in the industry and generally considered as an afterthought. They presume that security testing would not give return on investment whereas in long run it saves millions and can become a hallmark of excellence. However, academically security testing cannot be perceived as an add-on activity but a separate, fully fledged and integral component to emphasize and focus on security aspect of applications. Now a day, people are talking about security testing throughout development life cycle. A proper mechanism is therefore essentially needed to carry out security testing in order to uncover security errors and defects in the application at the very outset.

\footnotetext{
*Author for correspondence
} 
Security Testing can be broadly categorized into manual, automatic and hybrid of both depending on complexity and requirement of the software. Manual testing includes testing of software without using any automated tool or any script. It turns out to be a costly affair because of human effort intensive testing each and every aspect of software, whereas automatic methods of security testing can significantly reduce the cost of testing and increase the robustness of software by enabling thorough testing. In particular, automation of test data generation can improve testing productivity and will also lead to higher accuracy. This is because an automation tool can follow a sequence of steps faster than a person, and it can execute the tests overnight to present the results in the morning. Automatic security testing range from static testing to dynamic testing. Static testing includes testing of software without executing the software, like code reviews or static analysis while dynamic testing ensures testing by executing the software. Each of these techniques has their own merits and demerits. Static technique can be applied to very large program but it may generate false positive or spurious warning, while dynamic testing highlights actual errors but it may miss several errors due to missing certain path for testing.

Software industry is using different automatic testing techniques that include static, dynamic or combination of both however the main challenges include optimal test case generation and complete path coverage so that the program testing should not miss any path. Hence, complete path testing is an essential activity to uncover several safety and security issues, which are not very feasible by merely running the application with a set of input parameters. Researchers and practitioners propose several techniques for testing the program automatically and to tackle these issues.

Random testing ${ }^{5-7}$ and symbolic testing ${ }^{8-11}$ are widely used automatic testing techniques. Random testing technique does not need to know internals of programs and are very easy to follow but fails to explore search space in a structured way. It may fail to explore important paths (i.e., path to which special cases of input data can lead to an execution) and it may also explore the same program path repeatedly. On the other side, symbolic testing is a promising technique that thoroughly executes the program symbolically taking into consideration each and every path. Although, it suffers from limitations notably includes dealing with native calls, floating point computations and dealing with complex constraints. These limitations are the noteworthy challenges before research community. Available literature shows that significant amount of research is done in the area and researchers and practitioners are working hard to find the solutions to these limitations but it still remains amongst the debatable issues.

Besides all these limitations, symbolic execution is very effective for security testing ${ }^{9-12}$ as it verifies each and every path in the program and assures that no untested path is left out. This approach automatically detects the corner cases where programmer fails to allocate memory or manipulate buffers, which may lead to security vulnerabilities ${ }^{11}$. In this paper, we use advantage of symbolic execution as it traverses each and every path to execute different pre injected exceptions. We model different environment failures such as server down, file not present etc through different injected exceptions because the creation of actual environment failure is very difficult and largely infeasible.

The paper is organized as follows: Section 2 describes the concept of symbolic execution. Section 3 highlights recent work related to security testing using symbolic execution. Section 4 discusses the vulnerabilities raised due to improper error handling. Section 5 presents the proposed framework Symexc. Section 6 demonstrates Algorithms and their implementation and section 7 discusses on results. Finally, section 8 concludes the paper.

\section{Symbolic Execution}

Symbolic execution is a program analysis and testing technique that symbolically executes the program instead of executing a program on a set of input ${ }^{13,14}$. Recently research community has shown significant interest and recognized it as one of the promising testing techniques. A number of practical tools based on symbolic execution have emerged in the recent past. Unlike concrete execution where path is determined by the inputs, symbolic execution executes the program for each and every path based on Path Condition (PC). Each branch point has its own path condition and $\mathrm{PC}$ at higher level is the aggregation of current branch and the PC of previous level branch. Symbolic execution internally contains two components: (i) Path condition generation and (ii) path condition solver, which are briefly described as follows:

\subsection{Path Condition Generation}

In normal execution only one path gets executed because of either truth or falsify of a branch condition, but in 
case of symbolic execution both the paths are taken into consideration by generating path conditions for both true and false paths. Suppose an expression is given as if(cond) then $S 1$ else S2 then in this case PC for the true branch would be $P C \rightarrow P C \wedge$ cond and $\mathrm{PC}$ for false branch would be $P C \rightarrow P C \wedge \neg c o n d$. So in general the PC can be depicted as $P C\left(p_{i}\right) \rightarrow \Sigma P C\left(p_{i-1}\right) \wedge b_{i}$ where $p i$ is any branch in a program and $b_{i}$ is a expression in that branch. Here $i$ is an integer that takes value from a root branch to the branch for which we are interested to find $P C$.

\subsection{Path Condition Solver}

Symbolic execution uses Path condition solver or constraint solver for two purposes, first to check if path is feasible or not and second, to generate the inputs for the program that satisfies these constraints. Main challenge of symbolic execution lies here as many researchers agree that for the whole cost of symbolic execution, the cost of constraint solving dominates everything else since constraint solving is a NP-complete problem ${ }^{15}$.

Here we explain how PC for any branch can be calculated. Further, it is illustrated with the help of example program as depicted in listing 1 and its corresponding Symbolic execution tree in Figure 1. For every branch depicted in this figure, symbolic executor generates and solves constraints at run time. Like for the branch $\mathrm{X}<$
$\mathrm{Y}$, given in Figure 1, symbolic executor generates two constraints, $\mathrm{X}<\mathrm{Y}$ for true condition and $\mathrm{X}>=\mathrm{Y}$ for false condition.

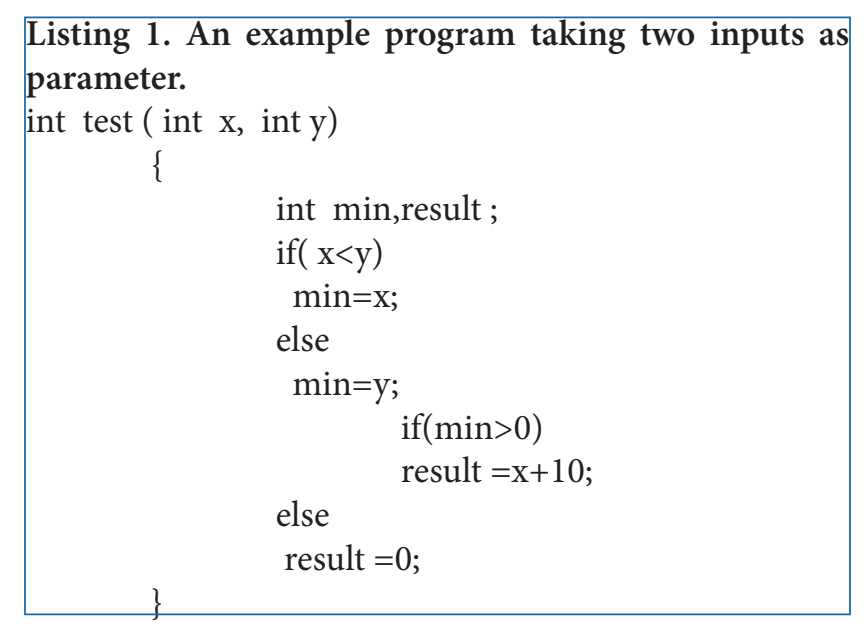

It is clear from the Figure 1 that there are four execution paths and each has its own corresponding path condition. Each path condition is aggregation of its previous and current path conditions. Although applying symbolic execution in program verification and test case generation is simple and straight forward but in actual practice, it poses some challenges ${ }^{16-20}$ for the complex and large real world software. A number of researches have been done to tackle these challenges and several solutions are proposed to mitigate them. Table 1 highlights some of

Table 1. Limitations of symbolic execution and their corresponding solutions

\begin{tabular}{|c|c|c|}
\hline Limitation & Brief description & Solutions \\
\hline Missing full path coverage & $\begin{array}{l}\text { Unable to explore full path due to complexity and large size } \\
\text { of the programs. }\end{array}$ & $\mathrm{Xie}^{17}$ \\
\hline $\begin{array}{l}\text { Inability in handling of com- } \\
\text { plex Mathematical functions }\end{array}$ & Problems in solving complex mathematical computations. & $\mathrm{CORAL}^{18}$, Borges et al. ${ }^{20}$ \\
\hline $\begin{array}{l}\text { Ineffective handling of exter- } \\
\text { nal functions }\end{array}$ & $\begin{array}{l}\text { Issues in solving path constraint that contains external func- } \\
\text { tions. }\end{array}$ & Summary $y^{22-24}$ \\
\hline $\begin{array}{l}\text { Inability in solving floating } \\
\text { point computations }\end{array}$ & $\begin{array}{l}\text { Generally, constraint solver approximate floating point num- } \\
\text { bers which could be a serious problem in certain systems like } \\
\text { real time system. }\end{array}$ & Botella et al. ${ }^{25}$, \\
\hline $\begin{array}{l}\text { Inability in solving string } \\
\text { constraint }\end{array}$ & $\begin{array}{l}\text { Several branch conditions include string comparison which } \\
\text { brings issues in constraint solving. }\end{array}$ & Hooimeijer and Weimer ${ }^{26}$, \\
\hline Object creation problem & $\begin{array}{l}\text { Test case for class methods includes the creation of an object, } \\
\text { change of its internal state, if required and at last invocation } \\
\text { of the required method which poses a great challenge because } \\
\text { sequence of method call is unknown or partially known. }\end{array}$ & $\begin{array}{l}\text { Tonella }{ }^{27} \text {, Suresh et al. }{ }^{28} \text {, } \\
\text { Xusheng et al. }{ }^{29}\end{array}$ \\
\hline $\begin{array}{l}\text { Handling of Environmental } \\
\text { conditions }\end{array}$ & $\begin{array}{l}\text { In real scenario our program may interact with several } \\
\text { external resources to read or write data items which may be } \\
\text { noisy and unreliable. How should we test these cases through } \\
\text { symbolic executor as symbolic execution works only on pro- } \\
\text { gram signature? }\end{array}$ & $\begin{array}{l}\text { Framework proposed in this } \\
\text { paper. }\end{array}$ \\
\hline
\end{tabular}


these challenges along with their corresponding solutions currently available in the literature.

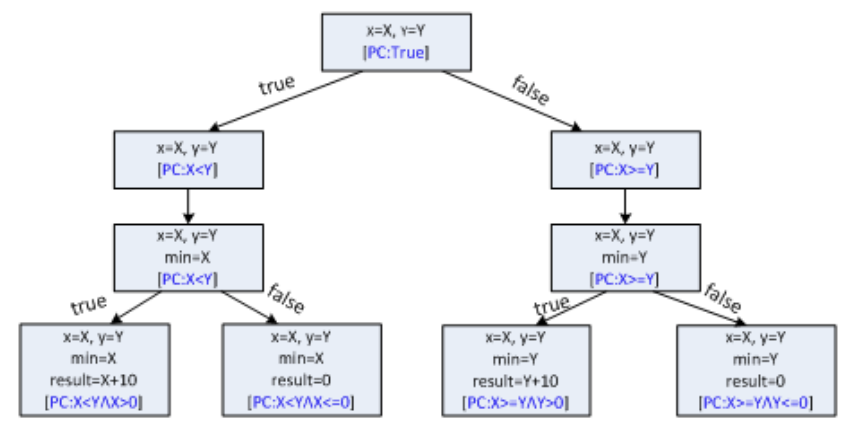

Figure 1. Symbolic execution tree of listing 1.

\section{Related Work}

Several methods and algorithms using Symbolic execution are available in the literature. It has been extensively used in research of Software testing and verification and in particular security testing ${ }^{8-12,18,20,21,30-35}$. This technique is being used in Industrial practices at many organizations notably Microsoft, IBM and NASA. It has also become an important part of several commercial products. Some of the popular methods related to symbolic execution in context of security testing are discussed as follows.

A directed automated random testing, $\mathrm{DART}^{9}$ has been proposed to automate unit testing using symbolic input. In order to execute and test a component in isolation with high path coverage, they proposed three techniques in sequence. First technique automates the extraction of interface of a program through which a program can obtain its input. Automatic generation of test driver for this interface is used at second stage and at last dynamic analysis is done to check how a program behaves on input generated at second stage and at the same time symbolic constraint for alternate path is generated. A method viz. $E X E^{10}$, an effective bug finding tool that ensures deep path testing of real code is proposed in the literature. EXE runs code on symbolic input instead of manually or randomly constructed input. When code conditionally checks symbolic expression, EXE forks execution so that it can explore each possibility. When a path terminates or hits a bug, EXE automatically generates input set that can actually hit the target by solving the path constraints through its own co-designed constraint solver, STP.

An effective security testing tool named $S A G E^{11}$ has been developed at Microsoft research lab that takes advantages of recent advancement in Symbolic execution and dynamic test generation. Their method generates the test cases very quickly unlike random testing, where chances of hitting a test case may be very minimal. This method effectively tests the corner cases of the program for which test cases generation is quite complex. In this paper author has claimed that several vulnerabilities can be found through their tool like ANI MS07-017 and others which are difficult to find otherwise. Furthermore, a method $K L E E^{12}$ which is a complete redesign of method EXE has been proposed by Cadar et al. KLEE is designed for robust and deep checking of broad range of applications and accommodates the lesson learnt from EXE live run. It includes variety of constraint solving optimization techniques and uses search heuristics to get high coverage.

Concolic (symbolic plus concrete) execution based method is proposed for high path coverage ${ }^{30}$. They compared and contrasted several search strategies which decides selection of a particular branch in each iteration for exploring path space. They also highlighted a key point that the number of execution paths required to get full branch coverage is bounded by the total number of branches in the program. A method to test particularly, object oriented programs by integrating evolutionary algorithm and dynamic symbolic execution proposed in the literature ${ }^{32}$. Dynamic symbolic execution is to generate special primitive-type arguments to cover the path, which are otherwise difficult to generate and evolutionary algorithm would generate the method sequence to lead the receiver object or non-primitive arguments to specific desirable state.

Several java based symbolic execution methods ${ }^{33,34}$ built on Java Path Finder (JPF) have been proposed by the research community. Among these, $j F U Z Z^{33}$ is a concolic whitebox fuzzer, built on top of JPF that executes the program both concretely and symbolically. jFUZZ systematically generates path constraint for each of true and false branch of any condition and queries constraint solver to generate input for the program. Thus, $j F U Z Z$ can systematically explore every control-flow path. Symbolic Path Finder $(\mathrm{SPF})^{34}$ combines symbolic execution with constraint solving and model checking for automated test case generation. SPF handles inputs and operations on Booleans, integers, real and complex data structures. It uses the generic constraint solver for integer, linear and interval arithmetic solver. Recently, first symbolic execution based automatic test tool generation for $\mathrm{C}++$ programs has developed and named it KLOVER ${ }^{35}$. They have extended the $\mathrm{C}$ based symbolic execution tool, KLEE 
and built their tool on top of KLEE. Their method has adopted variety of optimizations which are in addition to optimizations used in KLEE.

Although, a number of security testing methods based on symbolic execution have been proposed in the literature, but these all are unable to handle environmental condition failures or we can say these methods are not meant to handle these conditions or surely inadequate at handling this. Environmental condition failures can be exploited by attackers to crash the application. In this paper, we are using symbolic execution technique to handle these failures which may crash the application.

\section{Improper Error Handling}

Exception handling is a structured way to handle abnormal conditions in objects oriented code. Error handling in object oriented programming differs from traditional error handling in several ways such as centralize exception handling, keeping apart the error handling code from the normal code which makes reading and writing the code easier and so on. Despite all these advantages, improper error handling may emanate into several security issues including the following:

\subsection{Information Leakage}

This vulnerability means to expose critical system data, program structure including call stack or debugging information that may support adversary to learn about the software and formulate an attack ${ }^{36}$. Application program can also leak internal information and one of the most prominent reasons is exposing internals through exception handling. Information can be leaked because of stack trace publish by Exception handler. It is quite a tedious job to test our application about this information leakage as there are several exceptions which cannot be recreated very easily like RemoteException that may occur because of a number of communications related exceptions during the execution of a remote method call, Similarly SocketException occurs to indicate that there is an error while creating or accessing a Socket.

\subsection{Unreleased Resource Vulnerability}

Unreleased Resource vulnerability occurs if the program fails to release a system resource ${ }^{37,38}$. As an example, consider storerequest method of the program depicted in listing 2 where three classes FileInput stream, DataInput stream and BufferedReader have been used to read the request file to a buffer. Even though close() methods have been used on the objects fstream, $b r$ and in of above stream classes immediately after reading the contents of the request text file, it may be possible that due to abrupt termination of the program, close() method calls are bypassed. One possible reason for the program control to skip the execution could be file read error which might occur because of file not present at required location. The other reason may be due to required memory unavailable in the main memory. In these cases the program flow jump to catch block and objects would remain open until operating system closes these objects after program termination. This issue would leak the information and also if not closed properly, java program may leak file descriptors which is a limited resource and in worst case program will not be able to open any new file.

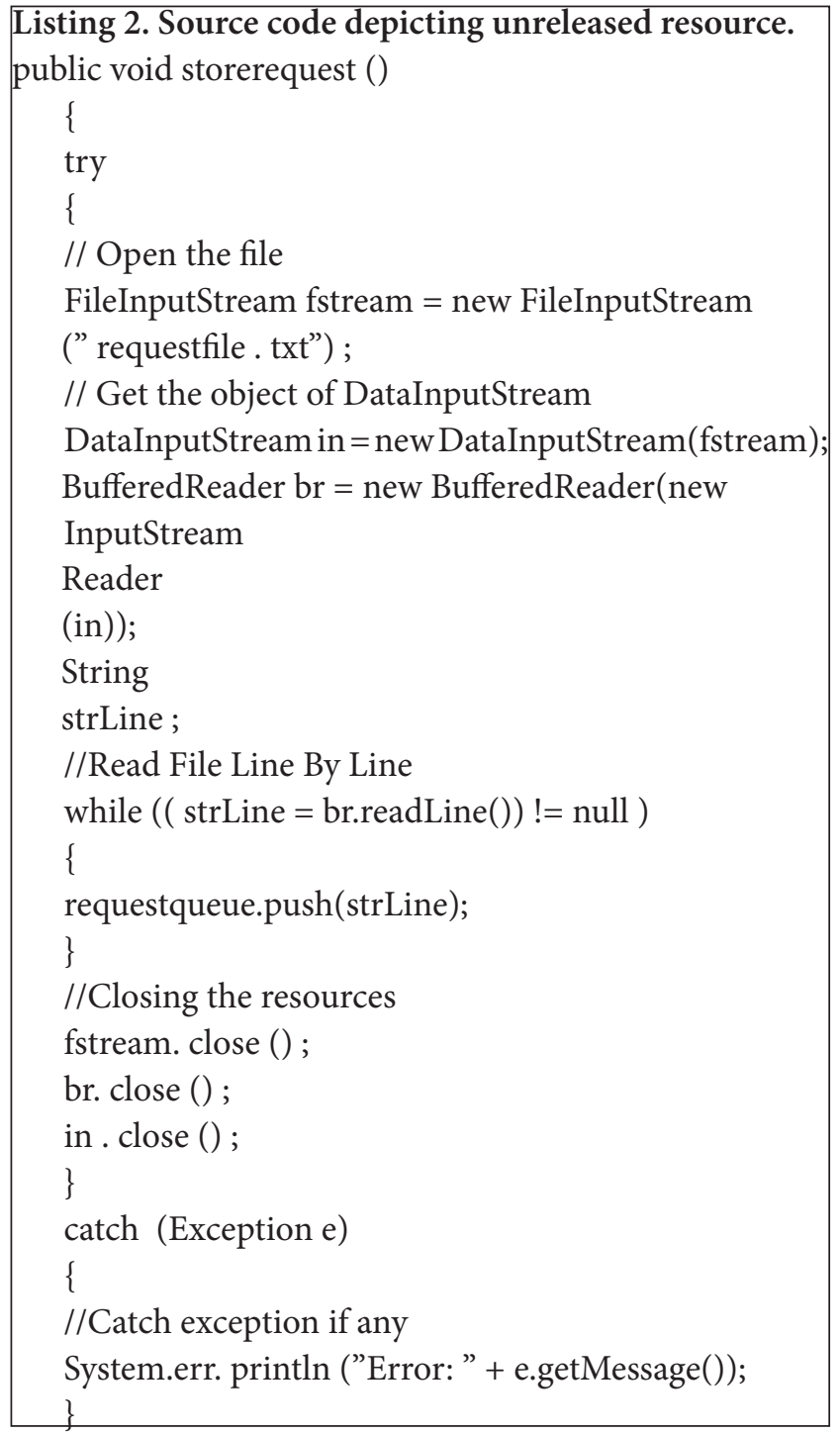




\subsection{Inconsistent State}

Throwing an exception can bring a program in an inconsistent state. Inconsistency could happen when a program throws an exception after manipulating an object and the object does not revert back to its previous state. This inconsistency can happen in multi threaded environment as well as in sequential programming. Inconsistency could be a serious issue as other thread would read inappropriate data which would result in application program corruption. Result of this inconsistency would be unpredictable; this could result in abrupt termination or illegal output. If an attacker intention is to corrupt the application then he would succeed in his intention by bringing the application to an inconsistent state.

\subsection{Program Crash (Denial of Service)}

If the exceptions are not caught properly, then this would cause the application to $\mathrm{crash}^{39}$. This could be a serious issue in multi threaded environment if exception is raised because of the shared object. Since every thread reads same state of shared object and hence each one will throw same exception. This would completely block the application. This type of vulnerabilities would allow remote attacker to crash the system. For example, an unhandled exception that causes a database server to crash could result in an effective denial of service for all its clients. If the attackers' motive was denial of service for the authorized users in the first case then he or she has actually succeeded.

Testing of the above said issues are not straight forward because several exceptions like remoteexception, socketexception and others cannot be recreated easily and also reaching those fault prone locations are not straight forward. Keeping in mind these two issues we proposed a framework that is discussed in section 5 .

\section{Proposed Framework}

The framework nicknamed as Symexc uses the concept of exception injector, symbolic execution and aspect oriented programming. It tests the program for security vulnerabilities caused by improper error handling. Symexc is expected to provide the following benefits for program security:

- It tests programs for errors where merely traversing program symbolically doesn't generate any error.

- It tests programs for program inconsistency, resource leakage and program crash in case of improper error handling.
- This method also tests the program for any information leakage during exceptions

Figure 2 shows the framework Symexc. Its actual working is summarized as follows: Firstly, the PUT passes through exception generator in order to generate all the possible exceptions in the program. Secondly, for all possible exceptions, aspects are created for generating actual exceptions at proper location during run time along with other aspects that are necessary to test the inconsistency and resource leakage in the program. PUT with these aspects is then executed through symbolic executor to hit those injected exceptions and finally a $\log$ is maintained to store resource leakage issues, inconsistencies and program crash.

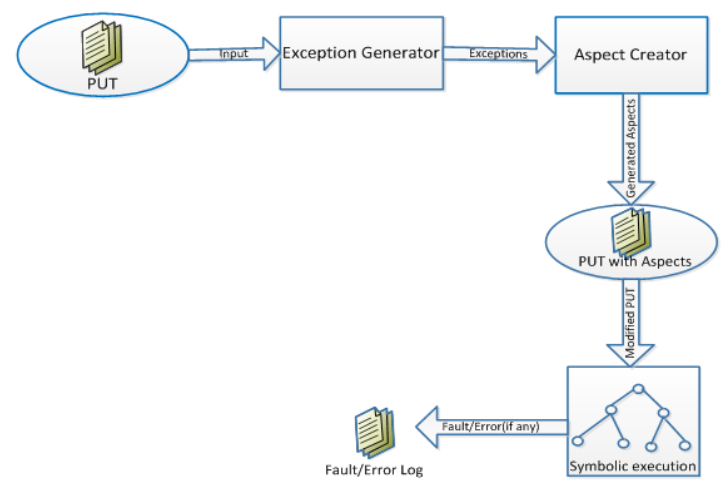

Figure 2. The framework, Symexc.

\section{Algorithms and Implementation}

This section briefly demonstrates algorithms and their implementations for the Symexc. Algorithm 1 calls modules to test program inconsistencies, resource leakage and Denial of Services. Algorithm 2 is developed to test program inconsistency and uses the concept of saving and comparing states before exception and function exit. Algorithm 3 is designed for resource leakage and program crash; different counters are used for different types of resources. These counters get incremented and decremented after each resource opening and closing respectively.

The method throws different injected exceptions (if there are any) during run time using different aspects. At the exit of point, we check if each counter used for different resources is settozero or not. If any of these counters is found to be non zero, it indicates that there is resource leakage issue in the program. Apart from resource leakage we 
also check whether program crashes due to run time exceptions generation. Program crash may happen when exception generator generates a non-checked exception which is not handled in the program.

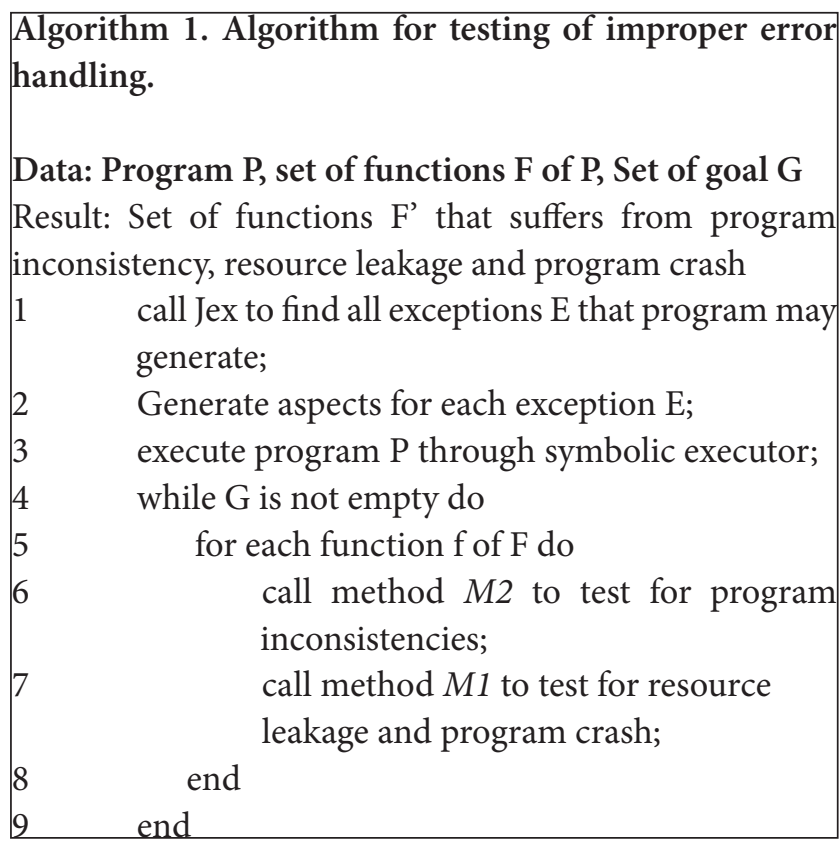

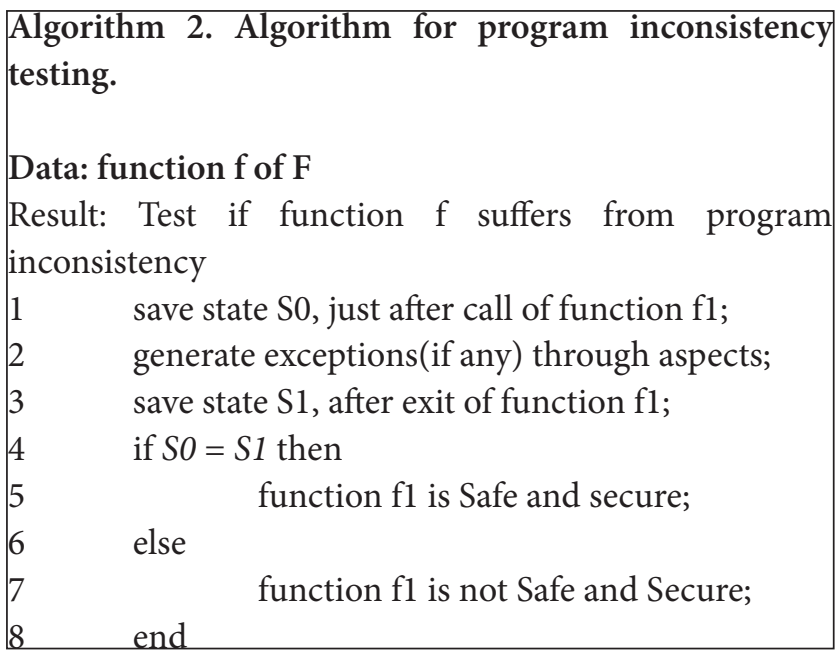

Algorithm 3. Algorithm for resource leakage and denial of services testing.

\section{Data: Program P}

Result: Test if program suffers from resource leakage and Denial of Services

2 set counter for each type of Resources;

3 set flag $=$ false;

4 Increase the counter during call of particular resource;

5 Decrease counter during close of each particular resource;
Generate exceptions(if any) through aspects;

Call an aspect during method exit; while Each counter do

\section{if counter! $=0$ then} set flag = true;

end

end

$$
\text { if } \text { flag }==\text { true then }
$$

PUT suffers from resource leakage; else

PUT does not suffer from resource leakage; end

Figure 3 shows how the interaction takes place among the components of Symexc, including the significant technical details. The tool is semi automated and its implementation details are given below:

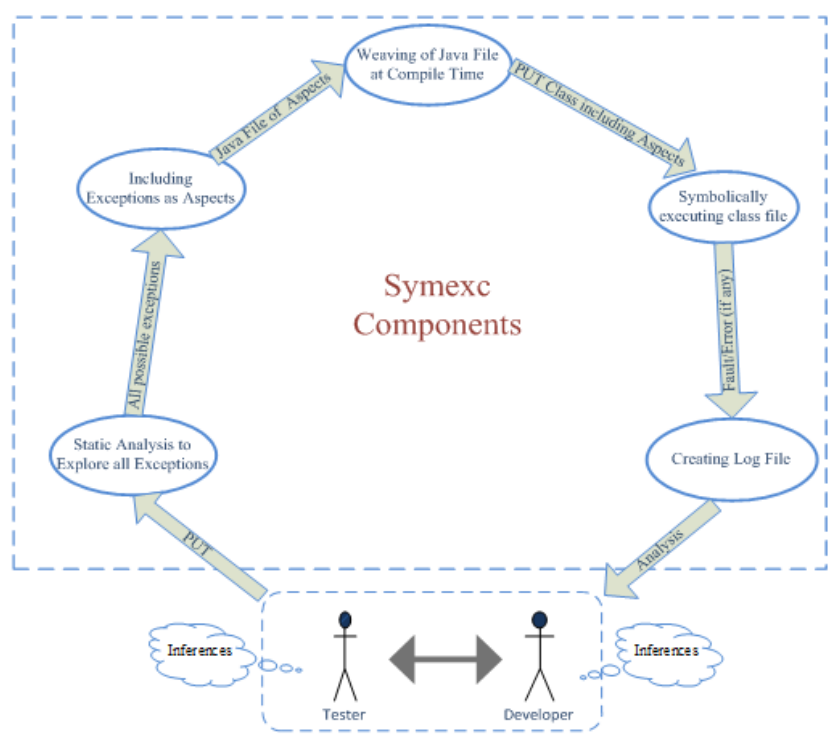

Figure 3. Interaction among Symexc components.

- Tester/developer gives PUT as an input which is analyzed to explore possible exceptions that the application may generate. Jex $x^{40}$ tool is used to statically analyze PUT and it finds out all actual exceptions that might arise at different points in the program including exceptions that are raised due to environmental conditions.

- Aspects for all these exceptions are incorporated or adjusted in the program using Aspectj ${ }^{41}$, an aspect oriented programming tool for Java. These aspects are then combined with the program as the part of PUT. 
- This modified PUT is symbolically executed to know error/faults in the program. Jcute ${ }^{42}$ is used as symbolic executor. It calls the library namely $\operatorname{lpsolve}^{43}$ to solve arithmetic inequalities. This library that can be called from any programming language and it can solve mixed integer linear programming constraints.

- Error/faults log is further analyzed to draw inferences or conclude on security issues and recommendations are made accordingly to the developers so that errors may be corrected and hence, the application is guarded from the issues well in advance.

\section{Discussion}

We have tested Symexc on some java packages/programs taken from open source java repositories ${ }^{41,44-47}$. Our selection criteria are to select those java programs which have some interaction with external interfaces. Programs shown in Table 2 are selected to test the proposed framework to ensure its validity to some extent. This table beside source shows attributes of package such as Line Of Code (LOC), number of classes and number of methods calculated using metrics plug-in in eclipse.

At very first stage, exceptions are generated for these programs through Jex tool which find all exceptions that might get raised at different program locations. First column of Table 3 shows exceptions generated for the program under test through Jex tool. At the second step, different aspects are created to throw runtime exceptions at different program locations. Apart from these aspects, some aspects are also created to implement our logic to test program inconsistency, information leakage and program crash. Second column shows the number of aspects for each program. Like in case of BytemanExample first two stages generate 23 numbers of exceptions and 28 numbers of aspects. In the third step, PUT is executed through symbolic path finder to symbolically execute the whole program so that each and every path gets executed. Last three columns of Table 3 shows the output of symbolically executing the programs for different

Table 2. Configuration of java packages tested on Symexc

\begin{tabular}{llccc}
\hline Package Name & Source & LOC & $\begin{array}{c}\text { Number of } \\
\text { Classes }\end{array}$ & $\begin{array}{c}\text { Number of } \\
\text { Methods }\end{array}$ \\
\hline SMTPMail & freesourcecode.net $^{5}$ & 252 & 03 & 08 \\
Bufferinout & Concurrency State Models \& Java Programs $^{2}$ & 89 & 05 & 14 \\
BytemanExample & Byteman Example Program $^{44}$ & 907 & 17 & 87 \\
Observer & Aspect5 $^{39}$ example java program & 89 & 6 & 13 \\
telecom & Aspect5 $^{39}$ example java program & 207 & 9 & 35 \\
coordination & Aspect5 $^{39}$ example java program & 120 & 7 & 22 \\
bean & Aspect5 $^{39}$ example java program & 79 & 2 & 8 \\
Chromosome Former & eToc Java program $^{6 x}$ & 928 & 1 & 35 \\
\hline
\end{tabular}

Table 3. Result of security testing of java programs

\begin{tabular}{|c|c|c|c|c|c|}
\hline Java Package Name & $\begin{array}{l}\text { Number of } \\
\text { exceptions } \\
\text { generated }\end{array}$ & $\begin{array}{c}\text { Number } \\
\text { of Aspects } \\
\text { generated }\end{array}$ & $\begin{array}{c}\text { Number of } \\
\text { Resource } \\
\text { Leakage }\end{array}$ & $\begin{array}{l}\text { Number of } \\
\text { Inconsistent } \\
\text { methods }\end{array}$ & $\begin{array}{c}\text { Number of } \\
\text { Program } \\
\text { crash(DoS) }\end{array}$ \\
\hline SMTPMail & 05 & 10 & 4 & 0 & 1 \\
\hline Bufferinout & 12 & 17 & 2 & 0 & 0 \\
\hline BytemanExample & 23 & 28 & 3 & 1 & 0 \\
\hline Observer & 3 & 8 & 0 & 0 & 1 \\
\hline telecom & 5 & 10 & 0 & 0 & 2 \\
\hline coordination & 6 & 11 & 0 & 1 & 1 \\
\hline bean & 20 & 25 & 2 & 0 & 0 \\
\hline ChromosomeFormer & 30 & 35 & 1 & 0 & 0 \\
\hline
\end{tabular}


programs under the categories of Number of resource leakages, number of inconsistent methods and number of program crashes. Like third stage generates three instances of resource leakage, one instance of inconsistent methods and two instances of program crashes.

It is very much evident with this experiment results that improper error handling is a serious issue in applications. Developers are not trained or usually forget/ exclude to handle exceptions properly. Resource leakages unlike program crash do not directly halt the system but indirectly may affect the whole system. Several cases of resource leakage have been observed in programs like SmtpClient.java program as shown in listing 3 of SMTPMail package. If an exception is raised at line no. 5 due to I/O error during creation of output stream, the control passes to catch block and instances of Socket and BufferedReader will remain open and hence resources will be leaked. Java 7 has introduced new feature Trywith- resources instead of try block that automatically closes all the resources included in Try-with-resources block. Although this feature is very effective in restricting resource leakage issue but a large volume of java code remains still present and in-use with old style try block.

Few instances of tested programs suffer from inconsistent state since the program do not revert the changes in case of exceptions like in the case of package coordination. Symexc also found some cases of program crashes since the program do not handle the exceptions properly. Program crash is a serious issue since it completely halts the system and authorized users can not use the services of system.

\section{Conclusion}

Symbolic execution is recognized as one of the most effective testing techniques for uncovering code vulnerabilities. It automatically detects corner faults where programmer generally fails to allocate memory or manipulate buffers, which leads to security loop holes. In this paper, we have used advantage of symbolic execution to traverse each and every path to execute different preinjected exceptions. We model different environmental failures such as server down, file not present etc through different injected exceptions because the creation of actual environmental failure is very difficult. The proposed framework Symexc, tests the program for improper error handling that may raise several security issues such as resource leakage, inconsistent state and program crash. The framework tests and verifies if such vulnerabilities exist in the program. Furthermore, the framework is implemented and validated on java programs and results were found to be highly encouraging.

\section{References}

1. Ganpati A, Sharma AK, Kalia A, Singh H. Relationship between line of code and complexity in open source software. 4th (ICACTE 2011) International Conference on Advanced Computer Theory and Engineering; ASME Press; 2011.

2. Khan FF, Mallika R. Analysis of various types of bugs in the object oriented java script language coding. Indian Journal of Science and Technology. 2015 Sep; 8(21). Doi: 10.17485/ ijst/2015/v8i21/69958.

3. Hydara I, Md Sultan AB, Zulzalil H, Admodisastro N. Removing cross-site scripting vulnerabilities from web applications using the OWASP ESAPI security guidelines. Indian Journal of Science and Technology. 2015 Nov; 8(30):1-5.

4. HP. 2011 top cyber security risks report. Technical white paper, HP, 2011.

5. Claessen K, Hughes J. Quick check: a lightweight tool for random testing of Haskell programs. ACM Sigplan Notices. 2011 May 20; 46(4):53-64.

6. Csallner C, Smaragdakis Y. JCrasher: an automatic robustness tester for Java. Software: Practice and Experience. 2004 Sep 1; 34(11):1025-50.

7. Forrester JE, Miller BP. An empirical study of the robustness of windows NT applications using random testing. Proceedings of the 4th USENIX Windows System Symposium; 2000 Aug 3. p. 59-68.

8. Cadar C, Engler D. Execution generated test cases: how to make systems code crash itself. Model Checking Software. 2005 Aug 22; 3639:2-23.

9. Godefroid P, Klarlund N, Sen K. DART: directed automated random testing. ACM Sigplan Notices. 2005 Jun 12; 40(6):213-23.

10. Cadar C, Ganesh V, Pawlowski PM, Dill DL, Engler DR. EXE: automatically generating inputs of death. ACM Transactions on Information and System Security (TISSEC). 2008 Dec 1; 12(2):10.

11. Godefroid P, Levin MY, Molnar DA, et al. Automated whitebox fuzz testing. NDSS. 2008; 8:151-66.

12. Cadar C, Dunbar D, Engler DR. KLEE: unassisted and automatic generation of high-coverage tests for complex systems programs. OSDI. 2008 Dec 8; 8:209-24.

13. King JC. A new approach to program testing. ACM SIGPLAN Notices. 1975; 10:228-33.

14. King JC. Symbolic execution and program testing. Communications of the ACM. 1976; 19(7):385-94.

15. Chen T, Zhang X-S, Guo S-Z, Li H-Y, Wu Y. State of the art: 
dynamic symbolic execution for automated test generation. Future Generation Computer Systems. 2012.

16. Anand $S$. Techniques to facilitate symbolic execution of real-world programs [PhD thesis]. Georgia Institute of Technology.

17. Xie T, Tillmann N, de Halleux J, Schulte W. Fitness-guided path exploration in dynamic symbolic execution. IEEE/ IFIP International Conference on Dependable Systems \& Networks, 2009, DSN'09; IEEE; 2009. p. 359-68.

18. Souza M, Borges M, d’Amorim M, Pasareanu CS. Coral: Solving complex constraints for symbolic pathfinder. NASA Formal Methods; Springer; 2011. p. 359-74.

19. Kiezun A, Ganesh V, Guo PJ, Hooimeijer P, Ernst MD. Hampi: a solver for string constraints. Proceedings of the eighteenth international symposium on Software testing and analysis; ACM; 2009. p. 105-16.

20. Lakhotia K, Tillmann N, Harman M, De Halleux J. Flopsysearch-based floating point constraint solving for symbolic execution. Testing Software and Systems; Springer; 2010. p. 142-57.

21. Borges M, Amorim MD, Anand S, Bushnell D, Pasareanu CS. Symbolic execution with interval solving and meta-heuristic search. 2012 IEEE Fifth International Conference on Software Testing, Verification and Validation (ICST); IEEE; 2012 Apr 17. p. 111-20.

22. Godefroid P. Compositional dynamic test generation. ACM SIGPLAN Notices. 2007; 42:47-54.

23. Anand S, Godefroid P, Tillmann N. Demand-driven compositional symbolic execution. Tools and Algorithms for the Construction and Analysis of Systems; Berlin Heidelberg: Springer; 2008 Mar 29. p. 367-81.

24. Godefroid P, Nori AV, Rajamani SK, Tetali SD. Compositional may-must program analysis: unleashing the power of alternation. ACM Sigplan Notices. 2010; 45:43-56.

25. Botella B, Gotlieb A, Michel C. Symbolic execution of floating-point computations. Software Testing, Verification and Reliability. 2006 Jun 1; 16(2):97-121.

26. Hooimeijer P, Weimer W. Solving string constraints lazily. Proceedings of the IEEE/ACM international conference on automated software engineering; ACM; 2010. p. 377-86.

27. Tonella P. Evolutionary testing of classes. ACM SIGSOFT Software Engineering Notes. 2004; 29:119-28.

28. Thummalapenta S, Xie T, Tillmann N, De Halleux J, Schulte W. Mseqgen: Object-oriented unit-test generation via mining source code. Proceedings of the the 7th joint meeting of the European software engineering conference and the ACM SIGSOFT symposium on the foundations of software engineering; ACM; 2009. p. 193-202.

29. Xiao X, Xie T, Tillmann N, de Halleux J. Precise identification of problems for structural test generation. Proceedings of the 33rd International Conference on Software Engineering; ACM; 2011. p. 611-20.

30. Burnim J, Sen K. Heuristics for scalable dynamic test generation. Proceedings of the 2008 23rd IEEE/ACM interna- tional conference on automated software engineering; IEEE Computer Society; 2008 Sep 15. p. 443-6.

31. Pasareanu CS, Rungta N, Visser W. Symbolic execution with mixed concrete-symbolic solving. Proceedings of the 2011 International Symposium on Software Testing and Analysis; ACM; 2011. p. 34-44.

32. Inkumsah K, Xie T. Evacon: a framework for integrating evolutionary and concolic testing for object-oriented programs. Proceedings of the twenty-second IEEE/ACM international conference on automated software engineering; ACM; 2007. p. 425-8.

33. Jayaraman K, Harvison D, Ganesh V, Kiezun A. Jfuzz: A concolic whitebox fuzzer for java. NASA Formal Methods; 2009. p. 121-5.

34. Pasareanu CS, Rungta N. Symbolic pathfinder: symbolic execution of java byte code. Proceedings of the IEEE/ACM International Conference on Automated software Engineering; ACM; 2010. p. 179-80.

35. Li G, Ghosh I, Rajan SP. Klover: A symbolic execution and automatic test generation tool for $\mathrm{c}++$ programs. Computer Aided Verification; 2011. p. 609-15.

36. Whittaker JA. How to break software. Boston: Addison-Wesley; 2002.

37. Howard M, LeBlanc D, Viega J. 24 deadly sins of software security: programming flaws and how to fix them. McGraw-Hill, Inc.; 2009.

38. Natarajan M. Automated source code analysis to identify and remove software security vulnerabilities: case studies on java programs. International Journal of Software Engineering. 2013 Jan; 06(01):3-32.

39. Graff M, Van Wyk KR. Secure coding: principles and practices. O’Reilly Media, Inc.; 2003.

40. Robillard MP, Murphy GC. Static analysis to support the evolution of exception structure in object-oriented systems. ACM Transactions on Software Engineering and Methodology (TOSEM). 2003; 12(2):191-221.

41. Laddad R. Aspect $\mathrm{j}$ in action: enterprise AOP with spring applications. Manning Publications Co.; 2009.

42. Sen K, Agha G. Cute and jcute: Concolic unit testing and explicit path model- checking tools. Computer Aided Verification. 2006; 4144:419-23.

43. lp solver. 2016 Mar 5. Available from: http:|http://lpsolve. sourceforge.net/

44. Barehttp java web server. 2016 Mar 5. Available from: https://www.savarese.org/software/barehttp/

45. Bounded buffer demonstration. 2016 Mar 5. Available from: http://www.doc.ic.ac.uk/ jnm/book/book_applets/ BoundedBuffer.html

46. Byteman example program. 2015 Dec 5. Available from: https://developer.jboss.org/wiki/FaultInjectionTestingWith Byteman\#where_can_i_download_the_tutorial_sources/

47. Smtpmail. 2015 Dec 12. Available from: http://freesourcecode. net/javaprojects/13639/SMTP-Mail-Client-Java- example\#. Vpj2n_196M9. 